\title{
Driver Fatigue Eeg Fuzzy Entropy Feature Analysis Based on Sliding Window
}

\author{
Zhendong Mu \\ The Center of Collaboration and Innovation, Jiangxi University of Technology, Nanchang, Jiangxi \\ Province, 330098, China
}

Keywords: EEG(Electroencephalogram); Fatigue Detection; Sliding Window; Fuzzy Entropy

\begin{abstract}
For the non-stationary signal, entropy is a good method of analysis, now has been successfully applied to the study of driver fatigue detection feature, now for the driver fatigue detection method is based on the piecewise independent samples, it is difficult to describe the gradual process of fatigue, based on fuzzy entropy as feature extraction method. In 1 sampling periods for the window, using 1/10 cycle steps, the collected EEG signals were analyzed by continuous, sliding window fuzzy features describe the gradual process of driver fatigue.
\end{abstract}

\section{Introduction}

With the popularity of private cars, people travel more and more dependent on cars, single driving time is longer and longer, followed by driving fatigue caused by more and more accidents, The National Highway Traffic Safety Administration(NHTSA) conservatively estimates that 100,000 police-reported crashes are the direct results of driver fatigue each year. These crashes result in an estimated 1,550 deaths and 71,000 injuries, annually . Fatigue driving was reportedly involved in $16 \%$ of all crashes and over $20 \%$ of the crashes on highways . Therefore, it is very necessary to detect the state of fatigue in the process of driving in time .

In the EEG signal analysis method, entropy analysis method has been successfully used in many areas of research, such as the use of different research focus on the picture identification differences are, by calculating entropy feature of EEG signals, using SVM classifiers to achieve feature analysis of individual differences in the characteristics of entropy. The same entropy feature was also used for the detection of driver fatigue, through the design of virtual driving environment, the state of fatigue induced by the driver in the virtual driving environment, then the driver fatigue and normal EEG signal is segmented and the formation of normal samples and fatigue samples, using fuzzy entropy, approximate entropy, sample entropy, entropy, wavelet a variety of entropy entropy method for feature extraction and analysis, finally realize the driver fatigue detection.

The fatigue is a gradual process, although the normal and extreme fatigue can realize the value of the two analysis results can be achieved with extreme fatigue state detection, reduce the traffic accidents caused by fatigue, but this method can well describe the driver's fatigue state of process, so easy to cause the miscarriage of justice, practical method is not high, the sliding window in the form of driver fatigue detection process, through the 1 sampling period for the window, using 1/10 cycle steps, continuous acquisition of EEG signal were analyzed by calculating the fuzzy entropy of EEG signal characteristics of each step, dynamic measurement of driver.

\section{Data Acquisition}

The EEG data are collected by the Brian-computer Interface Lab of Jiangxi University of Technology from university students (12 subjects: 8 male and 4 female, their average age is 21.5). Within 24 hours prior to the experiments, these subjects would take no tea or coffee and 8 hours sleep at night before the experiment is required. The subject is given an operation introduction while an electrode cap is put on his/her head. After the subject is familiar with driving in the road conditions, EEG signal collection begins. For a short time of driving, it is difficult to enter into a state of fatigue to produce reliable and effective EEG. Unfortunately, for a longer period of driving, most participants have uncomfortable and unpleasant feelings including boredom, testiness and nausea. Therefore, according to previous experience in fatigue related experiment, each subject is asked to drive for 40 minutes without break before taking a questionnaire to check the status, based 
on the Li's subjective fatigue scale and Borg's CR-10 scale. The questionnaire results show the subject was in driving fatigue. The experiments are authorized by Academic Ethics Committee of Jiangxi University of Technology.

With a 32-electrodes Neuroscan data acquisition device, the international 10-20 system is used for EEG collection protocol. All channel data were referenced to two electrically linked mastoids at A1 and A2, digitized at $1000 \mathrm{~Hz}$ from a 32-channel electrode cap (including 30 effective channels and 2 reference channels) based on the international 10-20 system and stored in a computer for the offline analysis [33-36].Eye movements and blinks were monitored by recording the horizontal and vertical EOG.

After the EEG signals collected, the main steps of data preprocessing was carried out by the Scan 4.3 software of Neuroscan. The raw signals were first filtered by a $50 \mathrm{~Hz}$ notch filter and a $0.15 \mathrm{~Hz}$ to $45 \mathrm{~Hz}$ band-pass filter to remove the noise.

\section{Feature Calculation Method}

1) set the window size, this article set 1000 sampling points for the window;

2) setting the step size, this paper sets each sliding window step to 100 sampling points;

3) calculate the fuzzy entropy,The procedure for the FuzzyEn-based algorithm is described in detail as follows:

1) Set a L-point sample sequence: $\{v(i): 1 \leq i \leq L\}$;

2) The phase-space reconstruction is performed on $v(i)$ according to the sequence order, and a set of m-dimensional vectors are obtained $(m \leq L-2)$. The reconstructed vector can be written as

$$
T_{i}^{m}=\{v(i), v(i+1), \cdots, v(i+m-1)\}-v_{0}(i)
$$

Where $i=1,2, \cdots, L-m+1$, and $v_{0}(i)$ is the average value described as the following equation:

$$
v_{0}(i)=\frac{1}{m} \sum_{j=0}^{m-1} v(i+j)
$$

3) $d_{i j}^{m}$, the distance between two vectors $T_{i}^{m}$ and $T_{j}^{m}$, defined as the maximum difference values between the corresponding elements of two vectors:

$$
\begin{aligned}
& d_{i j}^{m}=d\left[T_{i}^{m}, T_{j}^{m}\right]=\max _{k \in(0, m-1)}\left\{\left|v(i+k)-v_{0}(i)-\left(v(j+k)-v_{0}(j)\right)\right|\right\} \\
& (i, j=1 \sim L-m, i \neq j)
\end{aligned}
$$

4) According to the fuzzy membership function $\sigma\left(d_{i j}^{m}, n, s\right)$, the similarity degree $D_{i j}^{m}$ between two vectors $T_{i}^{m}$ and $T_{j}^{m}$ is defined as:

$$
D_{i j}^{m}=\sigma\left(d_{i j}^{m}, n, s\right)=\exp \left(-\left(d_{i j}^{m}\right)^{n} / s\right)
$$

where the fuzzy membership function $\sigma\left(d_{i j}^{m}, n, s\right)$ is an exponential function, while $n$ and $s$ are the gradient and width of the exponential function, respectively.

5) Define the function $\gamma^{m}(n, s)$ :

$$
\gamma^{m}(n, s)=\frac{1}{L-m} \sum_{i=1}^{L-m}\left[\frac{1}{L-m-1} \sum_{j=1, j \neq i}^{L-m} D_{i j}^{m}\right]
$$

6) Repeat the steps from 1$)$ to 4$)$ in the same manner, a set of $(m+1)$-dimensional vectors can be reconstructed according to the order of sequence. Define the function:

$$
\gamma^{m+1}(n, s)=\frac{1}{L-m} \sum_{i=1}^{L-m}\left[\frac{1}{L-m-1} \sum_{j=1, j \neq i}^{L-m} D_{i j}^{m+1}\right]
$$

7) The fuzzy entropy can be expressed as:

FuzzyEn $(m, n, s, L)=\ln \gamma^{m}(n, s)-\ln \gamma^{m+1}(n, s)$

In these four entropies, $\mathrm{m}$ and $\mathrm{s}$ are the dimensions of phase space and similarity tolerance, respectively. Generally, too large of a similarity tolerance will lead to a loss of useful information. The larger the similarity tolerance, the more information may be missed. However, if the similarity tolerance is under estimated, the sensitivity to noise will be increased significantly. In the present study, $m=2, n=4$ while $s=0.2 * S D$, where SD denotes the standard deviation of the time series. 


\section{Results and Analysis}

By using the method of EEG signals of subjects for feature calculation, as shown in Fig.. 1 entropy features in continuous time, Fig. 1 is the upper part of the entropy entropy feature of sliding window, the sliding window size is only 100, so only from the upper part of Fig. 1 is difficult to see the change of driver fatigue state entropy, Fig. 1 shows the original half sliding window feature change trend, from the lower half of Fig. 1 can be seen, the entropy change is caused by a jitter, the EEG signal is a non-stationary signal, but the overall trend, characteristics of the fuzzy entropy of EEG signal with the the increase of driving time, driver fatigue slowly appear, entropy is a general trend of rising.
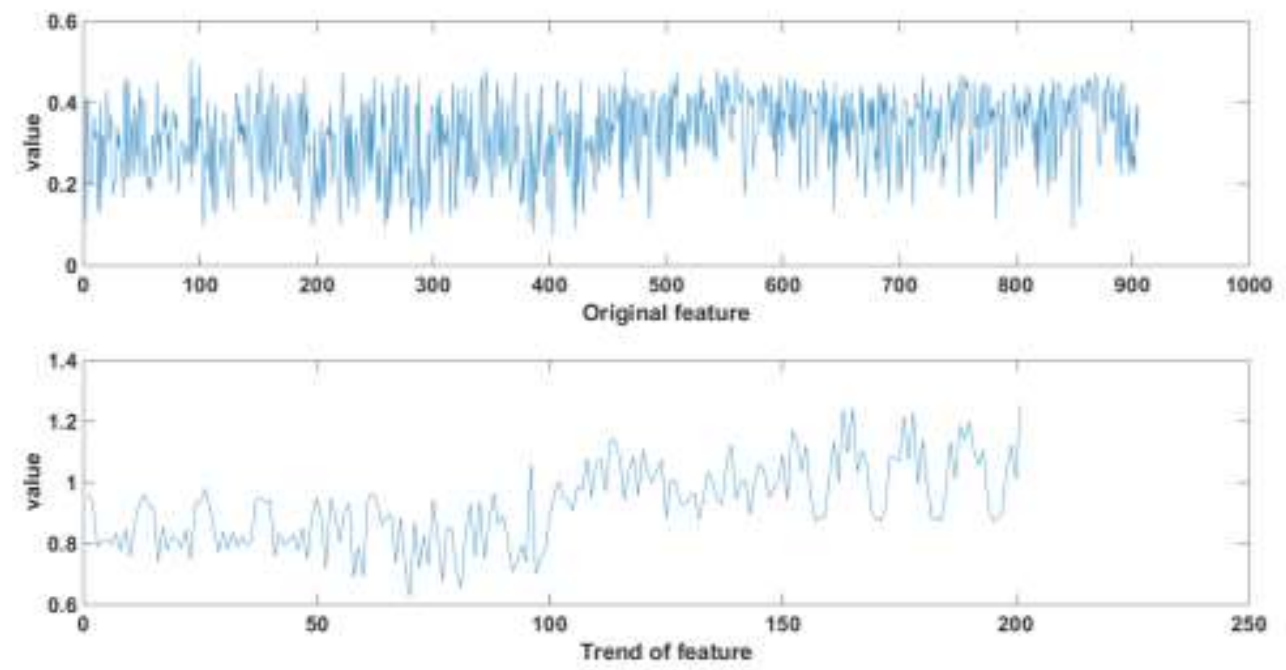

\section{Acknowledgments:}

This work was supported by Project of Science and Technology Department of Jiangxi Province (No. 20151BBE50079), and Natural Science Foundation of Jiangxi Province (No. 20142BAB207008). The Project of Science and Technology Department of Jiangxi Province (No. 20143BBM26048) also give us lots of supported.

\section{Reference}

[1]Liu C,and Subramanian R.Factors related to fatal singlevehicle run-off-road crashes[R],DOTHS811232,U.S. Department of Transportation,American National Highway Traffic Safety Administration, Washington,DC, USA,2009.

[2] Transport Research Laboratory - Driver fatigue-a killer on the roads [ EB / OL]. [1998-10-16]. http: // wwwntsb. gov/itsalprivate. htm.

[3] Annual statistical yearbook of road traffic accidents in People's Republic of China (2009) $[\mathrm{EB} / \mathrm{OL}](2010-05-17) \quad[2010-06-01]$. ht-tp://www. 122cn. com / read _ web. asp? Article ID $=3167$

[4] $\mathrm{Mu} \mathrm{Z}, \mathrm{Hu}$ J, Min J. EEG-Based Person Authentication Using a Fuzzy Entropy-Related Approach with Two Electrodes[J]. Entropy, 2016, 18(12):432.

[5] Deng B, Cai L, Li S, et al. Multivariate multi-scale weighted permutation entropy analysis of EEG complexity for Alzheimer's disease.[J]. Cognitive Neurodynamics, 2017, 11(3):217.

[6] Kaur R.; Singh K. Drowsiness Detection based on EEG Signal analysis using EMD and trained Neural Network. International Journal of Science and Research. 2013, 10, 157-161.

[7] Mousa Kadhim W.; Murugappan M.; Ahmmad B. Wavelet Packet Transform Based Driver Distraction Level Classification Using EEG. Mathematical Problems in Engineering. 2013, 3, 841-860.

[8] Correa A. G.; Orosco L.; Laciar E. Automatic detection of drowsiness in EEG records based on 
multimodal analysis. Medical Engineering and Physics. 2014, 36, 244-249.

[9] Resalat S.N.; Saba V. A practical method for driver sleepiness detection by processing the EEG signals stimulated with external flickering light. Signal, Image and Video Processing, 2015, 1-7.

[10] Yun K.; Park H. K.; Kwon D. H.; Kim Y. T.; Cho S. N.; Cho H. J. et al. Decreased cortical complexity in methamphetamine abusers. Psychiatry Research: Neuroimaging. 2012, 201, 226-232.

[11] Kumar S. P.; Sriraam N.; Benakop P. G.; Jinaga B. C. Entropies based detection of epileptic seizures with artificial neural network classifiers. Expert Systems with Applications. 2010, 37, 3284-3291. 